DOI: 10.1002/marc.((insert number))

\title{
Communication
}

\section{Power Density Threshold for Switching Off the Tack Adhesion of Colloidal Nanocomposites ${ }^{\mathrm{a}}$}

Ben S. Cooper, Robert S. Gurney, Elodie Siband, Damien Dupin, and Joseph L. Keddie*

B.S. Cooper, Dr. R.S. Gurney, Prof. J. L. Keddie

Department of Physics, University of Surrey, Guildford, Surrey GU2 7XH, United Kingdom E-mail: j.keddie@surrey.ac.uk

Dr. E. Siband

Allnex, Anderlecht Str. 33, B-1620 Drogenbos, Belgium

Dr. D. Dupin

Dpto. Nuevos Materiales, IK4-CIDETEC, Parque Tecnológico de San Sebastián, Paseo

Miramón, 196, 20009 Donostia - San Sebastián, Spain

Colloidal nanocomposite adhesives are made by blending soft adhesive particles with hard nanoparticles that sit at the particle boundaries to create a percolating structure. When the nanocomposite is heated with infrared (IR) radiation, the nanoparticles sinter together to create a rigid structure that hardens the composite and thereby switches off the tack adhesion. We have discovered that the IR power density of an irradiation for $20 \mathrm{~s}$ must exceed a threshold value of $1.07 \mathrm{Wcm}^{-2}$ before the tack is switched off. At lower power densities, our analysis of the sintering of the nanoparticles shows that there is not sufficient time to link them together into a rigid structure. Our results reveal that the switching of colloidal nanocomposite adhesives can be easily controlled through the IR power density and the time of the exposure.

\footnotetext{
${ }^{a}$ Supporting Information is available online from the Wiley Online Library or from the author.
} 
Published in Macromolecular Chemistry and Physics (2014) 215(10):998-1003

\section{FIGURE FOR ABSTRACT}

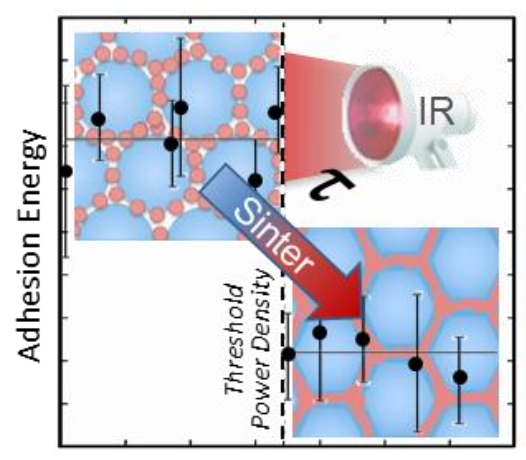

Power Density 


\section{Introduction}

Pressure-sensitive adhesives are permanently tacky at their temperature of use. ${ }^{[1,2]}$ They adhere to a variety of surfaces as a result of surface wetting that enables non-covalent bond formation, such as van der Waals and acid-base interactions. ${ }^{[3]}$ The ability to switch off the tack adhesion on demand has numerous applications, such as in the recycling of bonded components and in the painless removal of wound dressings. ${ }^{[4,5]}$ Research over the past decade has developed several strategies for achieving a switch-off of adhesion. The switch-off can be triggered by UV light to crosslink a soft polymer, ${ }^{[6-8]}$ by a temperature rise to switch liquid crystalline polymers from the smectic ${ }^{[9]}$ (or nematic ${ }^{[10]}$ ) state to the isotropic state, or by melting small molecules at the adhesive surface. ${ }^{[4]}$

In our previous research, ${ }^{[11]}$ we described nanocomposite adhesives that were created from blends of soft polymer particles and hard (glassy) nanoparticles. With relatively high evaporation rates during film formation, particles accumulated at the air interface. ${ }^{[12,13]}$ Because of the large size differential (size ratio of approximately 6:1), the nanoparticles assembled around the larger particles ${ }^{[14]}$ and created continuous percolating strings at the adhesive surface at relatively low concentrations of $15 \mathrm{vol} . \%$. The nanoparticles acted as mobile fillers that tuned the viscoelastic properties of the nanocomposite. ${ }^{[15-18]}$ When the nanoparticles were heated to temperatures above their glass transition temperature, $T_{\mathrm{g}}$, however, they sintered together to create a rigid skeletal structure, such as is illustrated in Figure 1. Subsequently, there was an increase in the elastic modulus accompanied by the emergence of a higher yield point. Whereas the initial nanocomposite exibited a measurable tack adhesion, the sintered nanocomposite lost its tackiness after heating and cooling back to room temperature. 

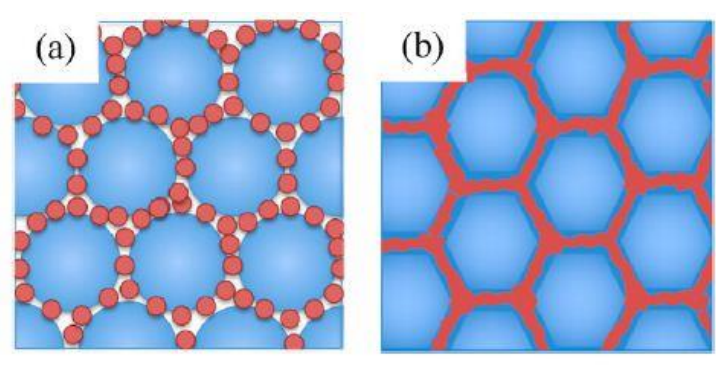

Figure 1: (a) at room temperature the hard NPs pack around the larger soft particles. (b) At temperatures above the $\mathrm{T}_{\mathrm{g}}$ of the NPs, a continuous network is formed due to the sintering of adjacent NPs. Image after Gurney et al.. ${ }^{[11]}$

Use of a mask for IR light used to heat the nanocomposites switched the adhesion off only in the unmasked regions. ${ }^{[19]}$ Thus, the nanocomposite adhesives were patterned via a simple process. In this initial work developing these responsive materials, the hard NPs were made from poly(methyl methacylate) copolymers having a $T_{\mathrm{g}}$ of $100{ }^{\circ} \mathrm{C}$. Below the $T_{\mathrm{g}}$, the nanoparticles are glassy and cannot sinter together, and hence the $T_{\mathrm{g}}$ defines a minimum switching temperature.

The switching mechanism requires the fusing together of the hard nanoparticles, via a sintering process, ${ }^{[20]}$ such that the percolating network of nanoparticles is able to support stress. In latex blends of hard and soft particles, Chevalier et al. reported that the elastic modulus increased by several orders of magnitude only when the hard particles were above the percolation threshold and when the temperature was raised above the glass transition temperature of the hard particles, so that they sintered. ${ }^{[21]}$ The driving force for the sintering of viscous particles is well understood. ${ }^{[22]}$ Particles flatten at their contact points to reduce their surface free energy. The energy reduction in flattening particles provides the energy for viscous dissipation. Frenkel ${ }^{[23]}$ considered the problem of two spheres each with a radius of $R$ and a viscosity of $\eta$ with a single contact point. He derived an equation for the contact angle as a function of sintering time, $t$. This simple analysis reveals that the characteristic time for 
particle sintering, $\tau$, is proportional to the viscosity but inversely related to the surface tension, $\gamma$, of the viscous liquid: ${ }^{[22]}$

$$
\tau \sim \frac{\eta R}{\gamma}
$$

Although Equation (1) captures the essential physics of the phenomenon, its use during a heating process requires a consideration of the temperature dependence, and hence the time dependence, of viscosity. During any heating process, the temperature $T$ is not a constant, but it increases over time. In turn, for a viscous polymer melt, the viscosity depends on temperature as given by the Williams-Landell-Ferry (WLF) equation ${ }^{[24]}$ :

$\eta(T)=\eta_{g} \exp \left[-\frac{34\left(T-T_{g}\right)}{80+T-T_{g}}\right]$

where the $\eta_{g}$ prefactor represents the viscosity at $T_{g}$, which is set equal to $6.5 \times 10^{10} \mathrm{~Pa}$ s here to be consistent with previous work. ${ }^{[25]}$ The value of the prefactor will not affect the temperature dependence of the viscosity, which is of greatest interest in this work.

In the present work, we use the same soft adhesive particles that we used previously. However, we introduce nanoparticles with a significantly lower $T_{\mathrm{g}}$ value of $34{ }^{\circ} \mathrm{C}$. This $T_{\mathrm{g}}$ is sufficiently high to avoid accidental switching at room temperature, but it is sufficiently low such that switching will be expected at temperatures that are only marginally above normal body temperature. The aim of the work is to understand how the switching off of the tack adhesion by a sintering method is a function of the time and temperature of heating. IR heating is used in our research, because it offers tight control of the temperature through adjustment of the power density. ${ }^{[26,27]}$ 


\section{Experimental Section}

\subsection{Materials}

Adhesives were made from blends of a "soft" poly( $n$-butyl acrylate) copolymer latex (called P2, hereafter) with a "hard" poly(methyl methacrylate-co- $n$-butyl acrylate) copolymer latex, called NP. The P2 latex synthesis included the use of a chain transfer agent (CTA) to reduce the mean molecular weight so as to impart more liquid-like properties to the adhesive matrix. The latex (P2) and nanoparticles (NP) were synthesized by emulsion polymerization by methods described elsewhere. ${ }^{[19]}$ The mean hydrodynamic diameters of the dispersed particles were determined by dynamic light scattering (photon correlation spectroscopy) using a Delsa Nano-C particle sizer (Beckman Coulter). Prior to the measurement, an aliquot of the aqueous dispersion was diluted in the range from 0.05 to $1 \mathrm{w} / \mathrm{w} \%$ using distilled deionized water (Milli-Q, $18 \mathrm{M} \Omega$ ).

The glass transition temperature of the fully-dried latex was found by differential scanning calorimetry (Q1000 TA Instruments, New Castle, DE, USA) at a heating rate of $10^{\circ} \mathrm{C} / \mathrm{min}$ in nitrogen. The $T_{g}$ was calculated using TA Instruments Universal Analyzer software, using the mid-point of the step in heat flow. The physical characteristics of the two latexes are presented in Table 1. It is apparent there that the NP particles are smaller than the P2 particles (with a size ratio of 1:4.5), and they have a higher $T_{\mathrm{g}}$. The weight-average molecular weights $\left(M_{\mathrm{w}}\right)$ and the polydispersity index $\left(M_{\mathrm{w}} / M_{\mathrm{n}}\right)$ are also listed in the table. The NP and P2 polymers both have a broad polydispersity, which is expected to broaden the glass transition region.

15 wt.\% NP latex (measured on the total solids mass) was blended with the P2 latex with thorough stirring. In preliminary studies, this concentration was found to be optimum for obtaining a large difference in the tack adhesion energy before and after heating. The addition of 
a greater proportion of NP led to a hardening of the composite and a consequent degradation of the initial adhesive properties, prior to switching.

The latex blends were cast onto cleaned glass substrates using a cube applicator with a $200 \mu \mathrm{m}$ gap to yield films with a thickness of $h_{\mathrm{o}}=100-200 \mu \mathrm{m}$ (measured with digital calipers). The films were dried at room temperature for at least 24 hours before testing.

Table 1. Characteristics of colloidal particles

\begin{tabular}{|c|c|c|c|c|c|}
\hline $\begin{array}{l}\text { Particle } \\
\text { name }\end{array}$ & $\begin{array}{l}\text { Hydrodynamic } \\
\text { diameter }^{(a)}[\mathrm{nm}]\end{array}$ & $\begin{array}{l}\text { Solids content } \\
\text { [wt.\%] }\end{array}$ & $\begin{array}{l}T_{\mathbf{g}}{ }^{(b)} \\
{\left[{ }^{0} \mathbf{C}\right]}\end{array}$ & $\begin{array}{c}M_{w} \\
{[\mathrm{~kg} / \mathrm{mol}]}\end{array}$ & $M_{w} / M_{n}$ \\
\hline $\mathrm{P} 2$ & 225 & $52 \pm 1$ & -34.2 & 301 & 36.1 \\
\hline NP & 50 & $37 \pm 1$ & 33.6 & 107 & 25.5 \\
\hline
\end{tabular}

${ }^{(a)}$ By dynamic light scattering; ${ }^{(b)}$ By differential scanning calorimetry

\subsection{Methods}

Nanocomposite adhesives were heated by a $4 \mathrm{~kW}$ carbon IR emitter (Heraeus Noblelight) with a length $0.7 \mathrm{~m}$. At its maximum power, the emitter has a temperature of $1200{ }^{\circ} \mathrm{C}$, which corresponds to a peak emission wavelength of $2 \mu \mathrm{m}$. The emitter has a very fast response time, reaching its maximum temperature within 1-2 s. The power density from the carbon IR emitter was measured using an optical power meter (Anritsu, ML910B) with a sensor for the near-IR range between $0.75 \mu \mathrm{m}$ and $1.8 \mu \mathrm{m}$ (Anritsu, MA9711A).

Measurements of the power density as a function of the emitter power and the distance from the lamp have been reported previously. ${ }^{[26]}$ In all experiments, the adhesive films were irradiated for $20 \mathrm{~s}$.

Probe-tack adhesion measurements were made by the Avery method using a commercial instrument (MicroSystems Texture Analyser, Godalming, UK). A $2.54 \mathrm{~cm}$ diameter spherical stainless steel probe made contact with the adhesive under a load of $4.9 \mathrm{~N}$ for $1 \mathrm{~s}$ before being withdrawn at a constant velocity of $0.1 \mathrm{~mm} \mathrm{~s}^{-1}$. This setting gives an initial strain rate of $1 \mathrm{~s}^{-1}$. 
At least five replicate measurements were made for each adhesive before and after exposure to IR radiation. The tack adhesion energy, $W_{a d h}$, was calculated after integration of the stress $(\sigma)$ strain $(\varepsilon)$ curves according to the equation:

$$
W_{a d h}=h_{o} \int \sigma(\varepsilon) d \varepsilon
$$

\section{Results and Discussion}

A range of IR power densities (from 0.4 to $1.7 \mathrm{Wcm}^{-2}$ ), which was obtained by varying the power of the lamp and its distance from the adhesives, was used during the heating of the nanocomposites. For each power density, the temperature of the adhesive was recorded as a function of time during a $20 \mathrm{~s}$ exposure. The temperature-time profiles are presented in Figure S1 (Supporting Information).

As a general trend, after heating with a lower power density, there was no significant change in the tack adhesion. On the other hand, after heating with a higher power density, the tack adhesion was effectively switched off. The probe-tack curves no longer showed a plateau corresponding to the drawing of polymer fibrils during debonding. The adhesive was no longer tacky to touch. Figure 2 shows some representative probe-tack curves, before and after IR heating.

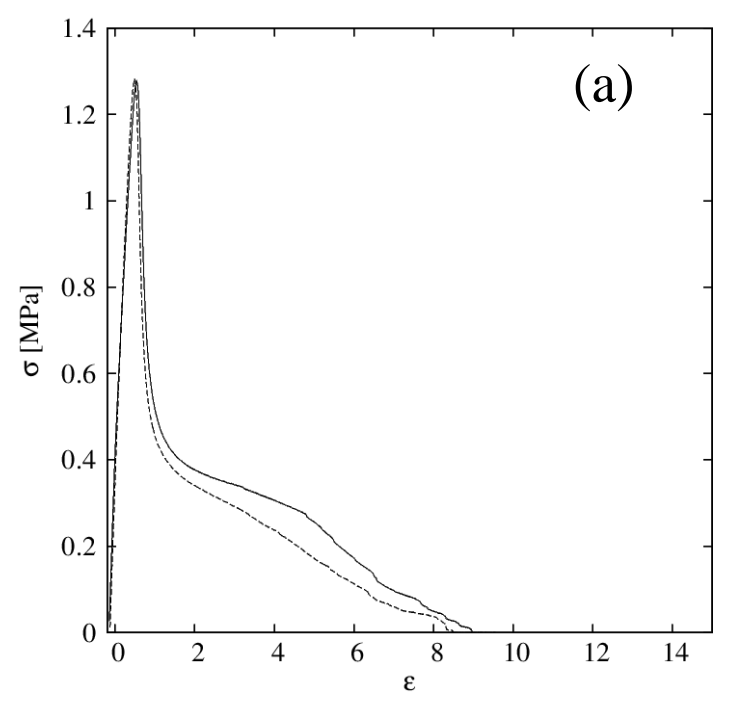




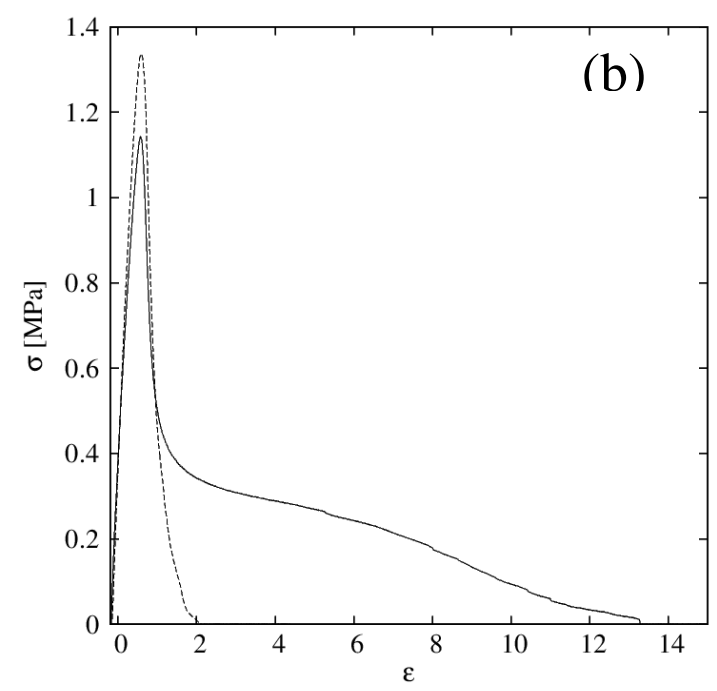

Figure 2. Representative probe-tack data for nanocomposite adhesives before (solid line) and after (dashed line) heating with IR radiation. (a) The emitter had a power of $2 \mathrm{~kW}$ and it was placed $4 \mathrm{~cm}$ from the adhesive for a low power density $=0.33 \mathrm{~W} \mathrm{~cm}^{-2}$. There is minimal change in tack. (b) The emitter had a power of $4 \mathrm{~kW}$, and it was placed $5 \mathrm{~cm}$ from the adhesive for a high power density of $1.3 \mathrm{~W} \mathrm{~cm}^{-2}$. The tack is lost.

Figure 3 provides a summary of the reductions in the adhesion energy, which are expressed as a percentage of the value prior to IR heating. Two regimes are seen in the data. With IR power densities of $1.07 \mathrm{Wcm}^{-2}$ or less, there is a negligible reduction in the adhesion energy. However above this threshold value, there is a reduction on the order of $30 \%$.

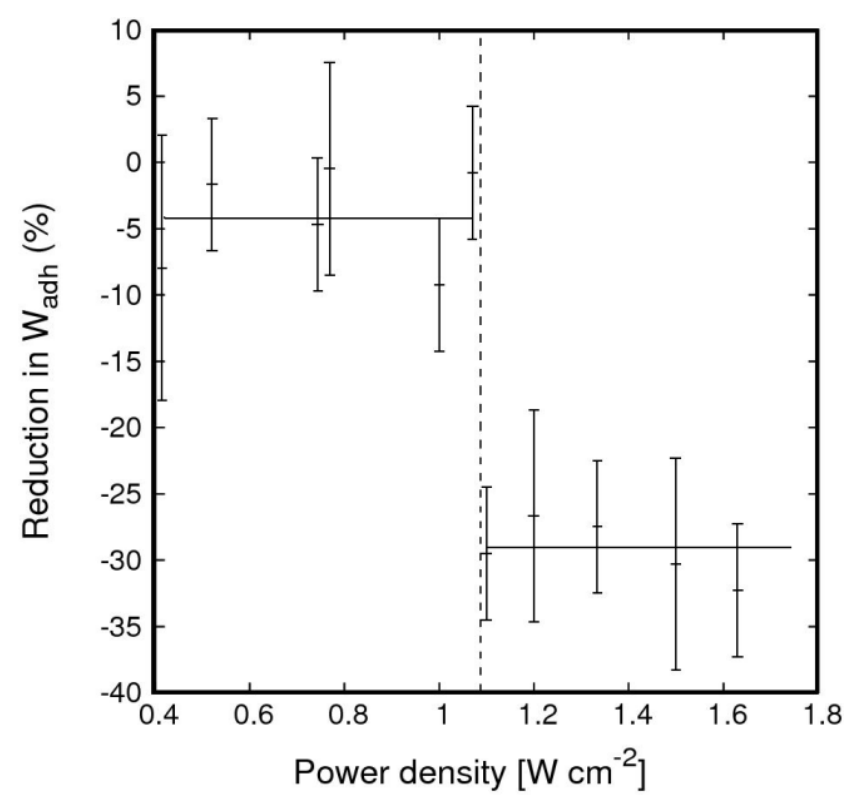


Figure 3. The reduction in the tack adhesion energy as a function of the IR power density after irradiation for $20 \mathrm{~s}$. Two regimes are seen, with a transition between 1.07 and $1.10 \mathrm{~W}$ $\mathrm{cm}^{-2}$

To understand why there is an abrupt "switch-off" of the adhesion energy above the threshold power density, rather than a more gradual loss of adhesion, we consider the characteristic times for sintering, $\tau$. We propose that the adhesion switch-off will not occur unless there is sufficient sintering between the nanoparticles to create a rigid skeletal structure. Hence, the characteristic time for sintering, $\tau$, must be shorter than the period of time at temperatures above the NP's $T_{\mathrm{g}}$.

For each experiment the polymer viscosity varies with time, because the temperature varies with time. We assume here that the viscosity of the NP copolymer follows the WLF dependence on temperature (Equation 2). The temperature-time data, $T(t)$, for each experiment was thus converted to viscosity-time $(\eta(t))$ curves. In the following analysis, we seek to use a single value of the NP polymer viscosity to represent its time average, $\bar{\eta}$. Accordingly, the $\eta(t)$ functions were integrated with respect to time and then divided by the total time of heating above the NP polymer $T_{\mathrm{g}}$ :

$$
\bar{\eta}=\frac{\int_{0}^{\tau} \eta(t) d t}{\int_{0}^{\tau} d t}
$$

where the lower limit of zero is set at the point when the polymer temperature reaches the $T_{\mathrm{g}}$, and $\tau$ represents the length of time at a temperature above the $T_{\mathrm{g}}$. Figure 4 (left axis) shows an inverse relationship between the time-averaged viscosity and the IR power density. In this analysis, it is expected that the temperature-dependence of viscosity is adequately captured by the WLF equation, but the magnitudes are not calculated with certainty because the value of $\eta_{\mathrm{g}}$ is only estimated. Using the estimated $\bar{\eta}$ for each of the power densities used, $\tau$ was calculated via Equation 1, using $R=25 \times 10^{-9} \mathrm{~m}$ and a typical value for the surface energy of 
a polymer, $\gamma=30 \times 10^{-3} \mathrm{Jm}^{-2}$. Values of $\tau$ are presented as a function of the power density in Figure 4 (right axis).

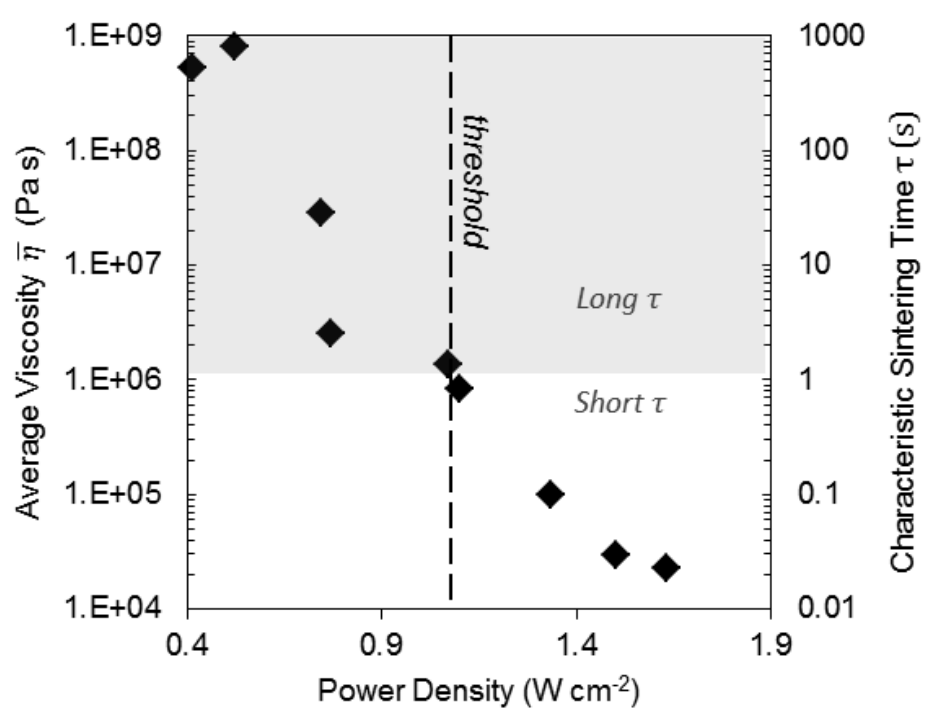

Figure 4. Time-averaged viscosity for experiments using a range of power densities of the IR emitter. The right axis shows the corresponding estimated characteristic sintering times, $\tau$. Tack adhesion was switched off at power densities above the threshold value of $1.07 \mathrm{~W} \mathrm{~cm}^{-2}$, where $\tau$ is shorter.

Consideration of the results in Figure 4 provides an important insight into explaining the existence of a threshold power density, above which the adhesion energy is switched off. We propose that at power densities above $1.07 \mathrm{Wcm}^{-2}, \tau$ is sufficienntly short so that the nanoparticles sinter together enough to create a rigid percolating network. The data in Figure 4 broadly support this argument. For instance, with a power density of $1.1 \mathrm{Wcm}^{-2}, \tau$ is estimated to be only $0.6 \mathrm{~s}$, which suggests sintering will be completed during the $20 \mathrm{~s}$ IR exposure. On the other hand, at a power density of $0.74 \mathrm{Wcm}^{-2}, \tau$ is estimated to be $24 \mathrm{~s}$, which is longer than the total IR irradiation time. Adhesion is not switched off in this case.

We have calculated the characteristic sintering time here using a time-average viscosity, $\bar{\eta}$. In our experiments, the heating time was fixed at $20 \mathrm{~s}$. We have not explored the effect of the total irradiation times, however we propose that $\bar{\eta}$ is the important parameter in 
determining the switch of the tack. Thus, if the temperature is raised and the exposure time shortened, or if temperature was lowered and the exposure time lengthened, we expect that the change in tack would be the same.

Finally, we note that a simple sintering model has adequately described the trends in our results. However, for the percolating structure of nanoparticles to have mechanical strength, polymer chain entanglements between adjacent particles are required [28]. Recent studies have indicated that interdiffusion and hence entanglements commence simultaneously with particle deformation for particles above $T_{\mathrm{g}}$ [29]. Cohesive particle contacts have been found elsewhere to develop via interdiffusion in polymer particles at temperatures just above their $T_{\mathrm{g}}$ even before the particles were fully compacted [30]. In this work, the chain reptation time is expected to be shorter than the time for the particles to deform to create particle/particle contacts. Hence, the particle deformation that is described by the sintering model is the rate-limiting step.

\section{Conclusions}

We have discovered that when switchable nanocomposite adhesives are heated for $20 \mathrm{~s}$ with IR radiation above a threshold power density of $1.07 \mathrm{Wcm}^{-2}$, the tack adhesion is switched off. Below the threshold value, there is little impact on the adhesion. That is, the transition is abrupt and controllable, making it more useful in applications. This result is explained by considering the characteristic time for the hard nanoparticles to fuse together in a sintering process. At power densities below the threshold value, the mean polymer viscosity is too high to enable sintering within the $20 \mathrm{~s}$ duration of the process. Our results provide an important insight into characteristics of switchable nanocomposite adhesives that will allow their future use without an inadvertent switch-off of the tack. The characteristic sintering time was found to be an important control parameter. 


\section{Supporting Information}

Supporting Information is available from the Wiley Online Library or from the author.

Acknowledgements: We thank Ron Swart, Keltoum Ouzineb, and Peter Mills for helpful discussions and Violeta Doukova (University of Surrey) for technical support. We acknowledge a PhD studentship for R.S.G. provided by Allnex, METRC, and the UK's Engineering and Physical Sciences Research Council.

Received: Month XX, XXXX; Revised: Month XX, XXXX; Published online:

DOI: 10.1002/marc.((insert number))

Keywords: adhesives, latices, nanocomposites, nanoparticles, sintering

[1] C. Creton, P. Fabre, in Mech. Adhes. (Eds.: D.A. Dillard, A. V Pocius), Elsevier, Amsterdamn, Netherlands, 2002, p. 535.

[2] D. W. Aubrey, in Handb. Adhes. 2nd Ed. (Ed.: D.E. Packham), Wiley-VCH, Weinheim, Germany, 2005, pp. 363-368.

[3] C. A. Dahlquist, in Treatise Adhes. Adhes. Vol. 2 Mater. (Ed.: R.L. Patrick), Marcel Dekker, New York, NY, USA, 1969, pp. 219-260.

[4] R. A. Chivers, Int. J. Adhes. Adhes. 2001, 21, 381.

[5] J. Klode, L. Schöttler, I. Stoffels, A. Körber, D. Schadendorf, J. Dissemond, J. Am. Acad. Dermatol. Venerol. 2011, 25, 933.

[6] J. M. Boyne, E. J. Millan, I. Webster, Int. J. Adhes. Adhes. 2001, 21, 49.

[7] I. Webster, Int. J. Adhes. Adhes. 1997, 17, 69.

[8] S. R. Trenor, T. E. Long, B. J. Love, J. Adhes. 2005, 81, 213.

[9] G. de Crevoisier, P. Fabre, J. M. Corpart, L. Leibler, Science (80-. ). 1999, 285, 1246.

[10] D. R. Corbett, J. M. Adams, Soft Matter 2013, 9, 1151.

[11] R. S. Gurney, D. Dupin, J. S. Nunes, K. Ouzineb, E. Siband, J. M. Asua, S. P. Armes, J. L. Keddie, ACS Appl. Mater. Interfaces 2012, 4, 5442. 
Published in Macromolecular Chemistry and Physics (2014) 215(10):998-1003

[12] C. M. Cardinal, Y. D. Jung, K. H. Ahn, L. F. Francis, AIChE J. 2010, 56, 2769.

[13] R. E. Trueman, E. Lago Domingues, S. N. Emmett, M. W. Murray, J. L. Keddie, A. F. Routh, Langmuir 2012, 28, 3420.

[14] H. Luo, C. M. Cardinal, L. E. Scriven, L. F. Francis, Langmuir 2008, 24, 5552.

[15] B. Pukanszky, F. Tudos, Makromol. Chem., Macromol. Symp. 1990, 221.

[16] D. Vidovska, F. H. J. Maurer, Compos. Interfaces 2006, 13, 819.

[17] A. Bellamine, E. Degrandi, M. Gerst, R. Stark, C. Beyers, C. Creton, Macromol. Mater. Eng. 2011, 296, 31.

[18] T. Wang, J. L. Keddie, Adv. Colloid Interface Sci. 2009, 147-148, 319.

[19] R. S. Gurney, D. Dupin, E. Siband, K. Ouzineb, J. L. Keddie, ACS Appl. Mater. Interfaces 2013, 5, 2137.

[20] A. Georgiadis, P. Bryant, M. Murray, P. Beharrell, J. L. Keddie, Langmuir 2011, 2176.

[21] Y. Chevalier, M. Hidalgo, J.-Y. Cavaillé, B. Cabane, Macromolecules 1999, 7887.

[22] R. E. Dillon, L. A. Matheson, E. B. Bradford, J. Colloid Sci. 1951, 6, 108.

[23] J. Frenkel, J. Phys 1945, 9, 385.

[24] R. G. Larson, The Structure and Rheology of Complex Fluids, Oxford University Press, USA, 1998.

[25] E. Gonzalez, M. Paulis, M. J. Barandiaran, J. L. Keddie, Langmuir 2013, 29, 2044.

[26] A. Georgiadis, F. N. Muhamad, A. Utgenannt, J. L. Keddie, Prog. Org. Coat. 2013, 76, 1786.

[27] A. Georgiadis, A. F. Routh, M. W. Murray, J. L. Keddie, Soft Matter 2011, 7, 11098.

[28] K.D. Kim, L.H. Sperling, A. Klein, B. Hammouda, Macromolecules, 1994, 27, 6841.

[29] K. Pohl, J. Adams, D. Johannsmann, Langmuir, 2013, 29, 11317.

[30] A. Turshatov, J. Adams, D. Johannsmann, Macromolecules, 2008, 41, 5365. 
Published in Macromolecular Chemistry and Physics (2014) 215(10):998-1003 
When hard nanoparticles in a nanocomposite adhesive are exposed to infrared (IR)

heating, they sinter together in a characteristic time, $\tau$. The adhesive is hardened, and the tack adhesion is switched off. For an IR exposure with a fixed time, this adhesion switch-off only happens when the power density is above a threshold value allowing the particles sufficient time to fuse.

B.S. Cooper, R. S. Gurney, E. Siband, D. Dupin, and J. L. Keddie*

Power Density Threshold for Switching Off the Tack Adhesion of Colloidal Nanocomposites

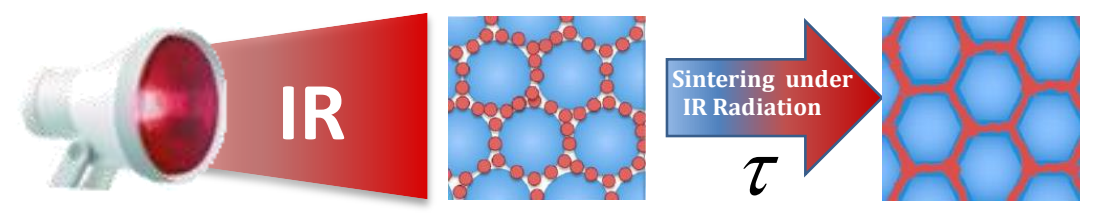


Copyright WILEY-VCH Verlag GmbH \& Co. KGaA, 69469 Weinheim, Germany, 2013.

\section{Supporting Information}

DOI: 10.1002/marc.2013\#\#\#\#\#

\section{Power Density Threshold for Switching Off the Tack Adhesion of Colloidal Nanocomposites}

Benjamin Cooper, Robert S. Gurney, Elodie Siband, Damien Dupin, and Joseph L. Keddie*

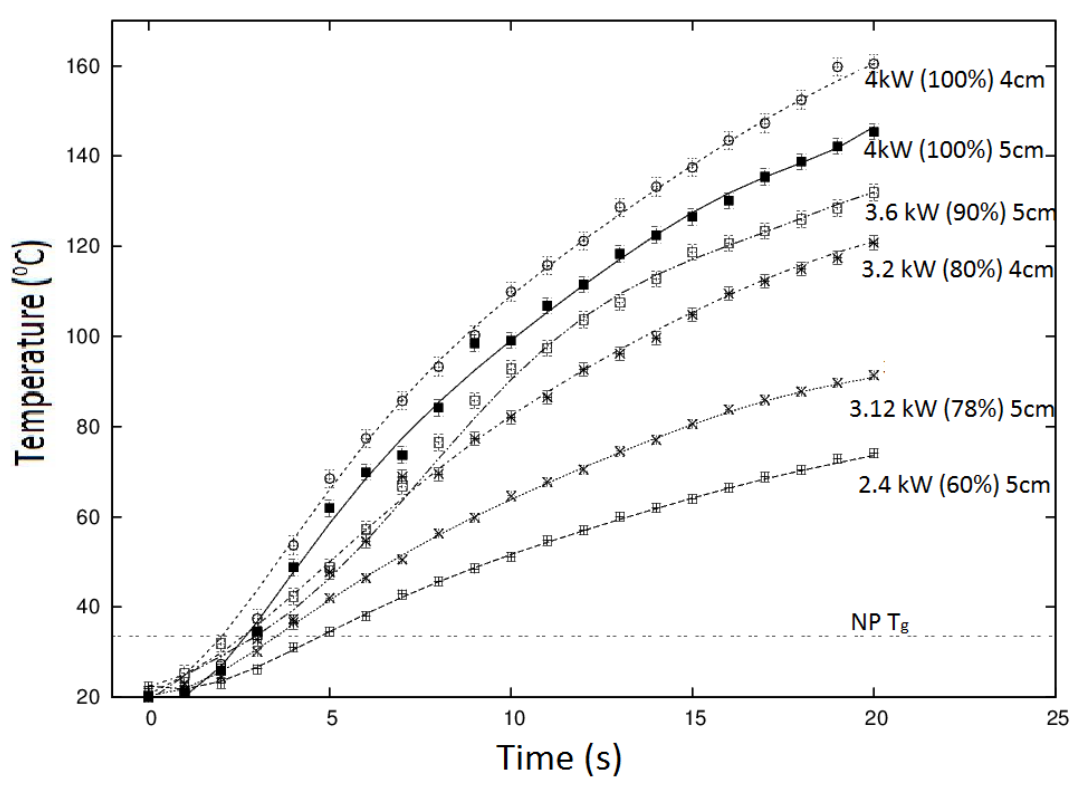

Figure S1. Heating profiles of adhesive films during irradiation with various IR lamp power levels (ranging between $2.4 \mathrm{~kW}$ and $4 \mathrm{~kW}$ ) and distances between the lamp and adhesives of either $4 \mathrm{~cm}$ or $5 \mathrm{~cm}$, as indicated. 